Abanico Veterinario. Enero-Diciembre 2021; 11:1-11. http://dx.doi.org/10.21929/abavet2021.25

Caso Clínico. Recibido: 28/01/2021. Aceptado: 01/06/2021. Publicado: 25/06/2021. Clave: e2021-9.

\title{
Contractura de tendones flexores en miembros anteriores de un ternero: reporte de caso
}

Flexor tendon contracture in the forelimbs of a calf: case report

\section{Agustín Fernández-Salas ${ }^{1 * ~ I D, ~ M i g u e l ~ A l o n s o-D i ́ a z ' ~ I D, ~ M a r i ́ a ~ M a r t i ́ n e z-F l o r e s ~}{ }^{2}$ ID, Daniel Morgado-Ramírez ID}

${ }^{1}$ Centro de Enseñanza, Investigación y Extensión en Ganadería Tropical, Facultad de Medicina Veterinaria y Zootecnia, Universidad Nacional Autónoma de México, Km. 5.5. Carretera Federal Tlapacoyan - Martínez de la Torre, C.P. 93600, Martínez de la Torre, Veracruz, México. ${ }^{2}$ Facultad de Ingeniería Agronómica y Zootecnia, Complejo Regional Centro, Benemérita Universidad Autónoma de Puebla, Carretera CañadaMorelos Km. 7.5, El Salado, C.P. 75460, Tecamachalco, Puebla, México. *Autor de correspondencia: Agustín Fernández-Salas. E-mail: mvz_salasuv@hotmail.com, alonsodma@hotmail.com, lupilla27mtz@hotmail.com, danielrmr1997@hotmail.com

\section{RESUMEN}

La contracción congénita de los tendones flexores en miembros anteriores es una de las anormalidades musculoesqueléticas más prevalentes en terneros neonatales. Esta alteración se puede presentar de forma leve, moderada y severa. El tratamiento de la condición leve y moderada puede ser mediante terapia farmacológica y fisioterapia; sin embargo, los casos severos se vuelven complejos por las lesiones secundarias en los terneros y la dificultad para que se alimenten, necesitando, en la mayoría de los casos, una intervención quirúrgica. El objetivo del presente reporte es describir el caso de un ternero con contracción severa de los tendones flexores de miembros anteriores y su corrección quirúrgica mediante tenotomía de los mismos. La cirugía se realizó con éxito y se reportan tiempos cortos de recuperación total mediante un protocolo postquirúrgico descrito a detalle en el presente documento.

Palabras clave: bovino, contractura, tenotomía, tendinopatía.

\begin{abstract}
Congenital flexor tendon contracture in forelimbs is one of the most prevalent musculoskeletal abnormalities in neonatal calves. This alteration can present in mild, moderate and severe forms. Treatment of the mild and moderate condition can be by pharmacological therapy and physiotherapy; however, severe cases become complex due to secondary lesions in the calves and difficulty in feeding, requiring, in most cases, surgical intervention. The objective of the present report is to describe the case of a calf with severe contracture of the forelimb flexor tendons and its surgical correction by tenotomy of the forelimb tendons. The surgery was successfully performed and short times of total recovery are reported by means of a postsurgical protocol described in detail in the present document.
\end{abstract}

Keywords: bovine, contracture, tenotomy, tendinopathy. 


\section{INTRODUCCIÓN}

Las patologías de las extremidades en el ganado bovino constituyen una de las preocupaciones económicas más importantes de los productores (Bruijnis et al., 2010), ya que influyen negativamente en su desempeño productivo y reproductivo, así como en el bienestar animal (Alsaaod et al., 2017). Los tendones son un elemento esencial de la unidad musculo-tendinosa de las extremidades, sirven de unión entre las fibras musculares y la superficie ósea y tienen la función de darle movilidad al hueso. De acuerdo a su localización, los tendones pueden presentar diversas características (Wavreille y Fontaine, 2009), tales como mayor flexibilidad y capacidad amortiguadora (para absorber golpes y limitar daño a los músculos) (Kirkendall y Garret, 1997). Las tendinopatías en los bovinos pueden ser causadas por inflamación, infecciones o alteraciones elásticas, siendo la contractura de los tendones flexores (CTF) de los miembros anteriores (articulación metacarpofalángica, del nudo o menudillo) una de las más frecuentes en crías bovinas (Weaver et al., 2018). Su presentación generalmente es bilateral, y raramente se desarrolla después del nacimiento (Duncanson, 2010). Las causas específicas de esta tendinopatía en los animales pueden deberse a deficiencias nutricionales de la madre durante la gestación, el mal posicionamiento de los fetos y/o problemas genéticos (Gaughan, 2017; Steiner et al., 2014). La CTF se clasifica en leve, moderada y grave. Los casos leves presentan una ligera flexión metacarpofalángica y los animales al desplazarse pisan con la punta de las pezuñas; en los casos moderados los terneros soportan, en varios lapsos de tiempo, su peso sobre esta articulación sobreflexionada; y en los casos severos, los terneros soportan todo su peso con la superficie dorsal de la articulación, pudiendo caer después de intentar incorporarse (Steiner et al., 2014; Weaver et al., 2018). Si no se tratan a tiempo los terneros con casos severos, se pueden desarrollar lesiones graves en la piel y las falanges, con probabilidad de desarrollar artritis supurante y rotura del tendón extensor.

El tratamiento de la CTF depende del grado de afectación. En los casos de contractura leve se debe asegurar la toma de calostro en las primeras horas de vida, y mantener al paciente incorporado la mayor parte del tiempo; el ejercicio fomentará la corrección progresiva de la alteración en estos casos (Divers y Peek, 2017). Se puede considerar el uso de una tablilla adherida a la suela de las pezuñas para estimular la extensión de los dedos. Se recomienda la aplicación de vitaminas del complejo B, minerales y pomadas rubefacientes junto con fisioterapia. En casos moderados, además del mismo tratamiento para los casos leves, se recomienda la colocación de una férula desde la cuartilla hasta la mitad del metacarpo o hasta la flexión del carpo. En casos severos que no responden a tratamientos terapéuticos conservadores, la corrección quirúrgica es el método de elección, siendo la tenotomía de los tendones flexores superficial, profundo o ambos, el método más utilizado (Sato et al., 2020). 
El objetivo del presente reporte es describir el caso de un ternero con contracción severa de los tendones flexores superficial y profundo de miembros anteriores y su corrección quirúrgica mediante tenotomía.

\section{Reseña}

\section{PRESENTACIÓN DEL CASO}

En una unidad de producción bovina (UPB) de Martínez de la Torre, Veracruz, México, se reportó el caso de un ternero macho 3/4 Cebú x Holstein de un mes de edad y $35 \mathrm{~kg}$ de peso, con imposibilidad para extender la articulación metacarpofalángica (nudo 0 menudillo) en ambos miembros anteriores. El ternero no caminaba de manera normal, frecuentemente presentaba un movimiento en retroceso, caídas y emaciación de una semana de evolución (Figura 1).
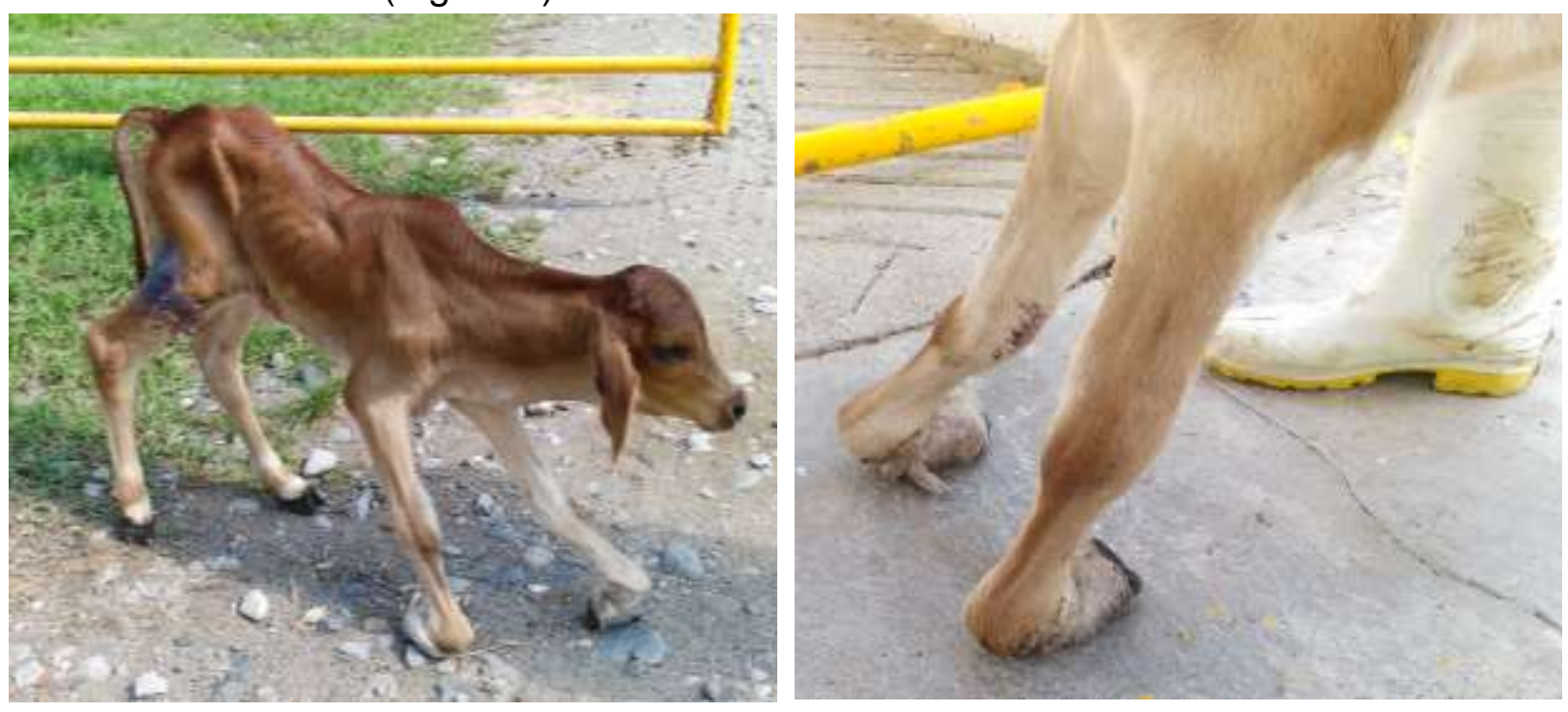

Figura 1. Desplazamiento de ternero con contractura de tendones flexores de miembros anteriores. Nótese la condición corporal y la alopecia en piernas

\section{Historia clínica}

\section{Anamnesis}

El ternero nació con la alteración de la articulación y hasta la tercera semana de edad se alimentó manualmente mediante biberón. A partir de la cuarta semana, el animal dejó de alimentarse adecuadamente y se observó emaciación progresiva. El ternero se incorporaba con menor frecuencia y se observaron lesiones sangrantes en la región dorsal de la cuartilla. Se menciona que se ha vendado el sitio de la lesión desde que inició la alopecia e inflamación de la zona (a partir de una semana de edad). Los tratamientos médicos previos incluyen la colocación de un entablillado provisional para estimular la extensión de la articulación, la administración de antiinflamatorios no esteroidales (diclofenaco y ácido tolfenámico), vitaminas ADE y del complejo $B$, antibióticos 
betalactámicos y ungüentos rubefacientes/antiflojísticos locales a base de salicilato de metilo, alcanfor y ácido carbólico por dos semanas, sin evolución positiva.

\section{Examen físico}

En la revisión clínica se detectó bajo peso, alopecia en piernas y vientre, así como lesiones en la región dorsal de la cuartilla (Figura 2). La articulación metacarpofalángica no se pudo extender de manera manual y se manifestó dolor a la manipulación. Durante la palpación, los tendones flexores digitales superficial y profundo de ambos miembros se percibieron contraídos y con tensión excesiva. Las constantes fisiológicas se presentaron dentro de rangos normales para la especie y edad (Piccione et al., 2010).

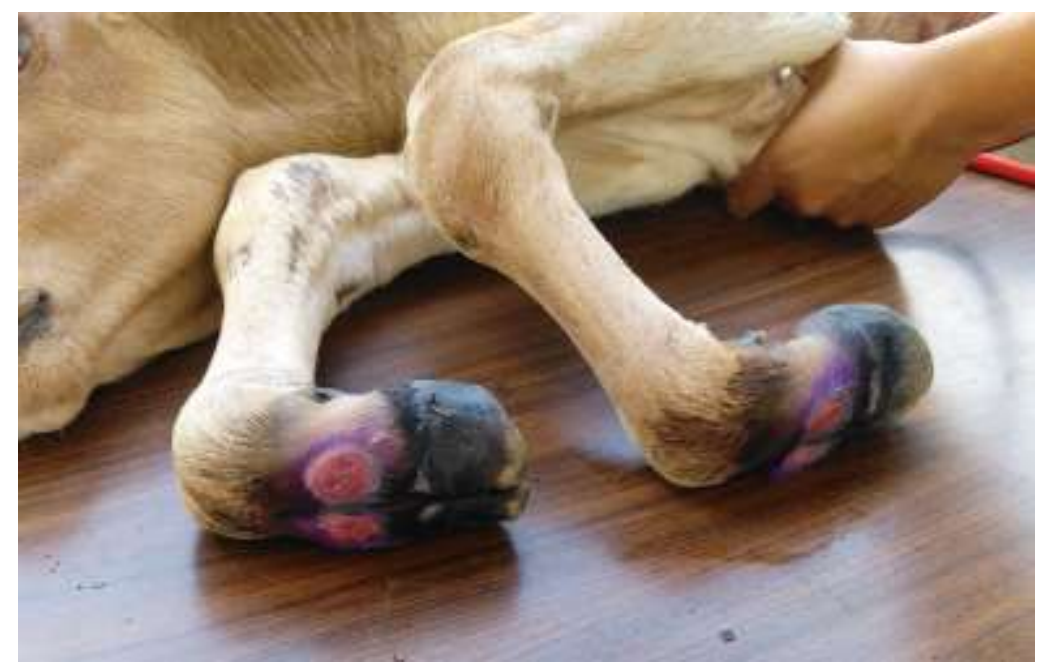

Figura 2. Lesiones en la región dorsal de la porción distal de los miembros anteriores en el ternero

\section{Diagnóstico}

Se determinó que el ternero presentaba contractura bilateral de tendones flexores digitales superficial y profundo en miembros anteriores. La alopecia en piernas y vientre era consecuencia del contacto prolongado con la orina acumulada en el piso debido al tiempo prolongado de postración. Se consideró que el tratamiento farmacológico y la terapia física ya no eran suficientes para la recuperación del ternero debido al severo grado de contracción y a las lesiones previamente mencionadas. Por lo tanto, se decidió la intervención quirúrgica mediante tenotomía de los tendones flexores digitales superficial y profundo.

\section{Abordaje terapéutico Consideraciones prequirúrgicas}

Se tomó una muestra de sangre del ternero para la realización de un hemograma, cuyo resultado estuvo dentro de rangos normales para la especie. Previo a la cirugía, el paciente fue restringido de alimento y agua durante 18 y 10 horas, respectivamente. 


\section{Procedimiento quirúrgico}

Se procedió a realizar la cirugía, iniciando con la sedación profunda del paciente con Xilacina $2 \%$ a dosis de $0.3 \mathrm{mg} / \mathrm{kg}$ de peso vivo por vía intramuscular (im). Esta dosis proporciona disminución del tono muscular y buena analgesia en bovinos. Se colocó al paciente en decúbito lateral, se realizó tricotomía de la región metacarpiana y se procedió a infiltrar localmente la zona con $20 \mathrm{ml}$ de lidocaína al $2 \%$. Se realizó antisepsia con yodo povidona y se llevó a cabo una incisión longitudinal de $6 \mathrm{~cm}$, desde el cuarto proximal del metacarpo hasta la mitad del mismo en la cara lateral para evitar la arteria palmar medial, justo sobre la posición anatómica de los tendones flexores superficial y profundo (Figura 3). Se abordaron cuidadosamente las fascias superficial y profunda para identificar y evitar dañar el nervio digital palmar lateral y las arterias y venas palmares metacarpianas. Se procedió a separar estas estructuras para protegerlas y localizar los tendones flexores superficial y profundo (Figura 4).

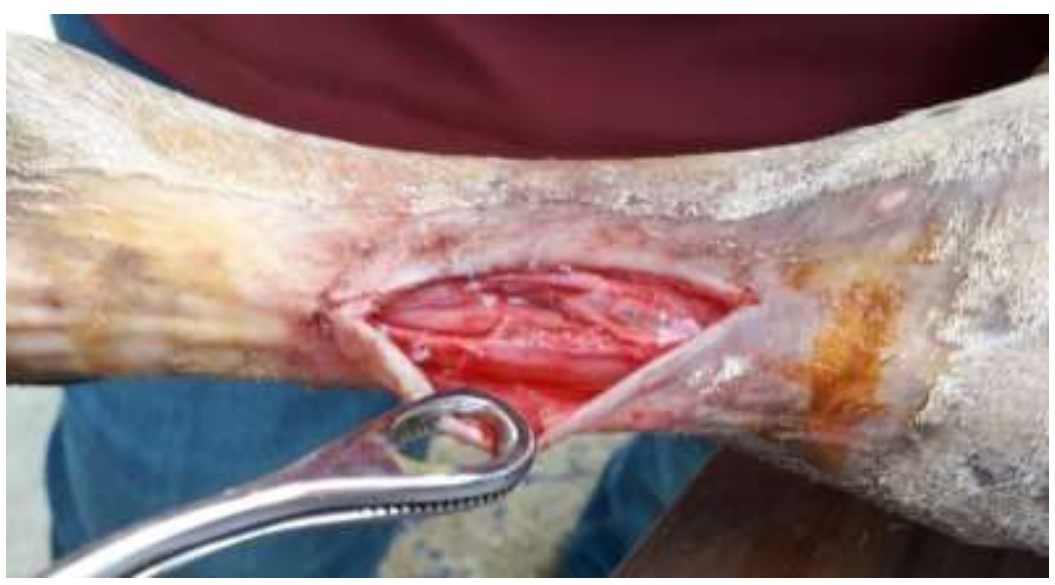

Figura 3. Incisión de piel y fascias para acceder a los tendones flexores digitales superficial y profundo

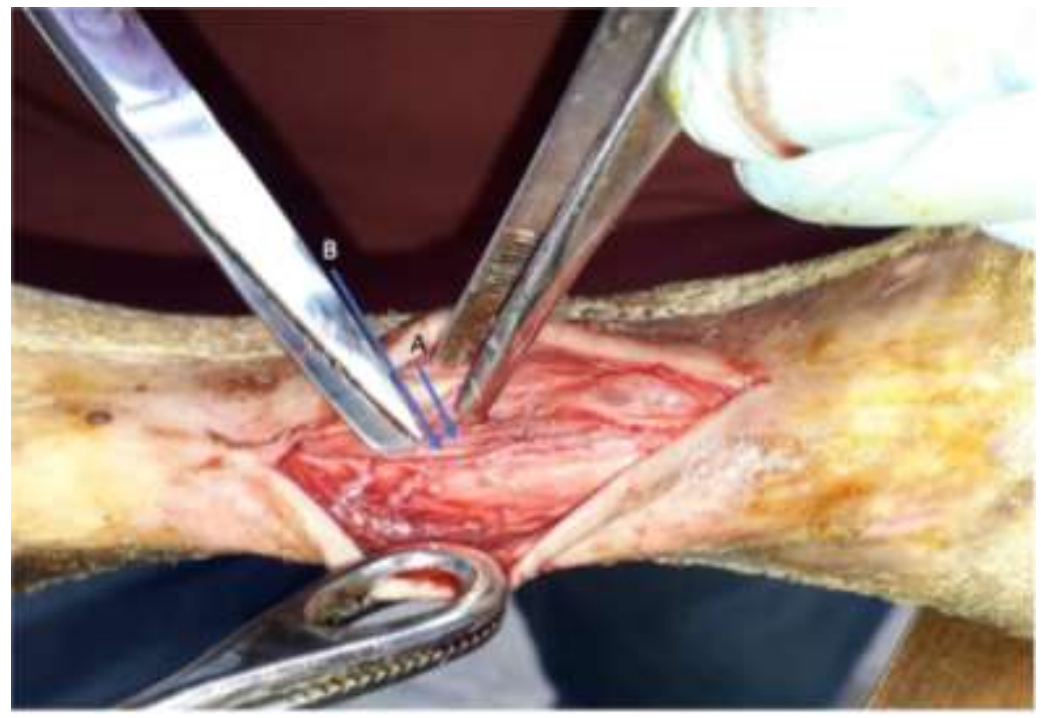

Figura 4. Identificación y protección de vasos sanguíneos metacarpianos (A) y nervio digital (B) 
Posteriormente, se elevó y extendió el tendón flexor superficial con la ayuda de una pinza de punta roma (Figura 5a) y se le realizó una transección quirúrgica con la ayuda de un bisturí (Figura 5b). Se realizaron movimientos extensores de la articulación metacarpofalángica, aunque mejoró un poco, aún era inadecuado el movimiento, por tanto, se procedió a elevar, tensar y exponer el tendón flexor profundo y se seccionó de la misma forma (Figura 5c). Es importante que la incisión del tendón profundo se realice en un sitio diferente del corte del tendón superficial para evitar la formación de adherencias.

Se corroboró la extensión y la mejoría fue evidente, por tanto, no hubo necesidad de mayor intervención sobre otra estructura. Se hidrató la zona con solución salina y antibiótico y se cerraron las fascias con sutura absorbible sintética calibre 3.0 en un patrón continuo. La piel se cerró con puntos separados simples con nylon \# 35. Se aplicó cicatrizante a base de aluminio micronizado, se vendó ligeramente la región falángica y metacarpiana, se extendió una capa de algodón y se colocó una férula en la región distalplantar, desde la corona de la pezuña hasta la porción proximal del metacarpo, para ayudar a la extensión de la articulación (Figura 6a). El mismo procedimiento se realizó en ambas extremidades.
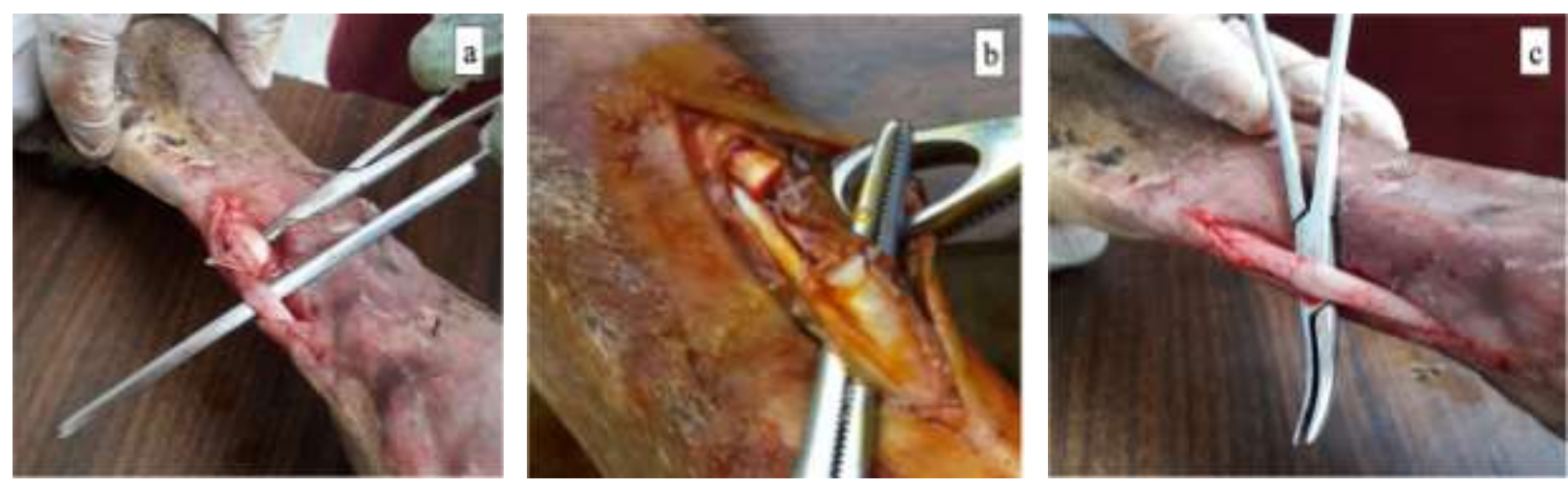

Figura 5. a) Presentación de tendones flexores superficial y profundo, b) disección de tendón flexor superficial y c) evaluación de función del tendón flexor profundo

\section{Tratamiento postquirúrgico}

Se administró antibiótico a base de penicilina G procaínica a dosis de 300000 UI cada $25 \mathrm{~kg}$ de peso (420 $000 \mathrm{UI}$ total) vía im durante tres días y flunixin de meglumine como antiinflamatorio y analgésico por 4 días a dosis de $1.5 \mathrm{mg} / \mathrm{kg}$ vía im. La férula se retiró cada tercer día para cambio de vendaje y tratamiento aséptico de la incisión y las lesiones prequirúrgicas mencionadas previamente. Las suturas de nylon en piel se retiraron 10 días después de la cirugía. 


\section{Evolución y resolución del caso}

Una semana después de la cirugía, el ternero manifestó mejor ánimo para ponerse de pie y empezó a colocar ligeramente la planta de las pezuñas. Dieciocho días después se dejó de colocar la férula y el paciente caminaba por sí mismo colocando la planta de las pezuñas la mayor parte del tiempo (Figura 6b). 30 días después, el ternero plantaba bien las pezuñas en el suelo, aunque caminaba con lentitud. Se dio de alta al ternero a los 35 días post cirugía, caminando lento, pero normal, sin manifestación de dolor o infección (Figura 6c).
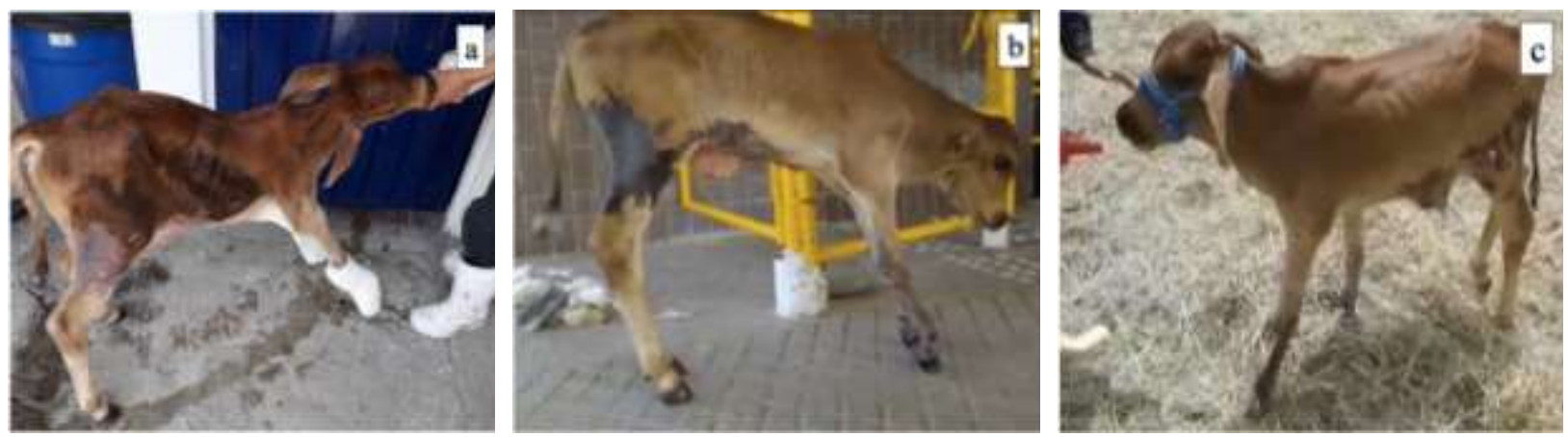

Figura 6. Evolución del ternero; a) férula para apoyar la extensión de la articulación metacarpofalángica, b) colocación de la planta de la pezuña sin férula, c) recuperación total con mejoramiento de la condición corporal

\section{DISCUSIÓN}

Dentro del grupo de las tendinopatías en bovinos, la contracción congénita de los tendones flexores es considerada como la anormalidad musculoesquelética más prevalente en terneros neonatales (Sato et al., 2020). Es importante considerar que, si no se tratan los terneros con casos severos, la piel y las falanges de las extremidades involucradas pueden lesionarse gravemente y aumentar la probabilidad de desarrollar artritis supurante, así como producir la rotura del tendón extensor digital como secuela (Figura 7). Debido a estas consecuencias, y a la dificultad de alimentarse por sí mismos, la mayoría de los casos terminan en el sacrificio del ternero.

En el presente caso se intervino a tiempo, ya que las lesiones secundarias estaban circunscritas en un área pequeña de piel, fueron superficiales y de fácil tratamiento (Figura 2). Evitar lesiones mayores, es clave para el éxito o fracaso de la cirugía (Steiner et al., 2014). En muchas UPB, los casos severos de esta deformidad no se tratan adecuadamente, debido a la renuencia o al desconocimiento de realizar la cirugía y al prolongado tratamiento post operatorio, sin embargo, como se puede observar, la técnica quirúrgica es bastante viable con los conocimientos anatómicos pertinentes de la zona. Uno de los principales objetivos en la implementación de este tipo de intervenciones quirúrgicas es acortar los tiempos de recuperación postoperatorios de los pacientes, para 
disminuir las probabilidades de lesiones por férulas y vendajes y para que los dueños de las UPB adopten estos procedimientos. Los tiempos de recuperación varían de 8 a 10 semanas en algunos reportes (Sohrt et al., 2013; Tamilmahan y Prabhakar, 2018), y en algunos casos, la lesión no se recupera totalmente (Sato et al., 2020). En este trabajo se acortó el tiempo de recuperación mediante fisioterapia, manejo farmacológico postoperatorio de la incisión y cambiando la férula y vendajes cada tercer día. Además, la aplicación de medicamentos solo se realizó la primera semana, y el ternero al mes ya era capaz de alimentarse por si mismo, coincidiendo con el reporte de Fernandes et al. (2020), quienes reportaron un mes de recuperación postquirúrgico. Sin embargo, es importante implementar y evaluar diversos protocolos postquirúrgicos, con diferentes razas, fármacos y grados de lesión, para ayudar a la toma de decisiones de las intervenciones adecuadas y promover el bienestar de los animales y su regreso a la vida normal.
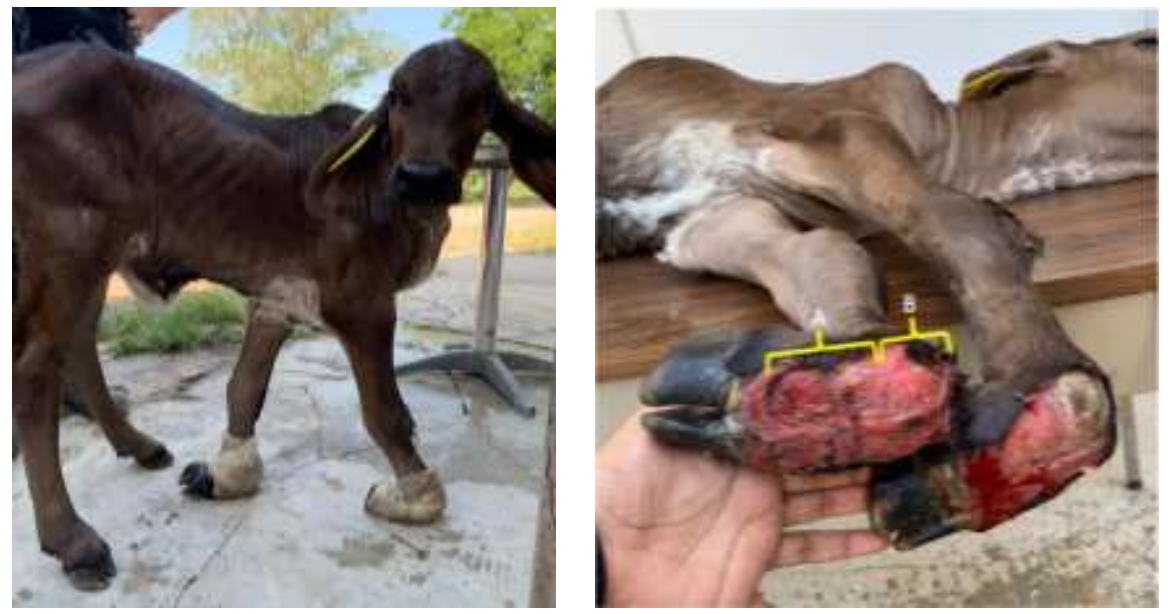

Figura 7. Lesión dorsal de la articulación de la cuartilla (A) y menudillo (B) en un caso severo no tratado de contractura de tendones flexores

La técnica quirúrgica utilizada para la tenotomía de los tendones en este reporte, demostró ser eficaz para este tratamiento y se disminuyó la invasión a los tejidos adyacentes a los tendones. En algunas otras especies, se ha recomendado la aposición de los extremos de los tendones post transección mediante una sutura lejos-cerca-cercalejos, con la finalidad de promover rapidez en la cicatrización (Fossum, 2019). Otros autores, en bovinos, han mencionado la incisión en " $Z$ " de los tendones para realizar una elongación del tendón como una propuesta para promover la recuperación rápida (Steiner et al., 2014), sin embargo, es importante considerar que los tendones son una estructura altamente inervada y el dolor postquirúrgico podría ser mayor. Se recomienda realizar un estudio comparando las tres técnicas para determinar la rapidez de la re-unión por fibroplasia y la recuperación de los animales. Hasta nuestro conocimiento, los reportes de casos clínicos que describan a detalle los protocolos para la realización de esta 
intervención quirúrgica en este tipo de tendinopatía son escasos (Tamilmahan y Prabhakar, 2018; Sato et al., 2020). Existen algunos reportes manejados con tratamientos conservadores en casos leves (Arieta y Fernández, 2011; Feito-Hermida et al., 2013), que en casos de contractura grave no serían suficientes. Otros reportes mencionan la intervención como parte complementaria de otras condiciones y en una sola extremidad (Sohrt et al., 2013).

Uno de los aspectos más importantes a considerar durante el tratamiento postoperatorio es la colocación de una férula adecuada, con bordes redondeados y protegidos, ya que una de las principales complicaciones postquirúrgicas es el desarrollo de úlceras o necrosis isquémica por presión del vendaje y/o la férula (Duncanson, 2010). En el presente reporte se diseñó una férula a base de tubo de PVC sin esquinas, con los bordes limados y una cubierta gruesa de algodón plisado sujeto con cinta adhesiva industrial. Esta férula protegió la piel y tan pronto como el ternero pudo pisar parcialmente con la planta de la pezuña, la férula se retiró para que el mismo peso del paciente promoviera la extensión de la articulación.

Finalmente, antes de decidir la implementación de esta cirugía, es importante realizar un adecuado diagnóstico de la alteración articular, ya que se debe diferenciar entre las diferentes formas de presentación de la contractura y descartar otras alteraciones como la artrogriposis (Fernandes et al., 2020; Divers y Peek, 2017), la cual es una forma extrema de contracción de tendones que se presenta en muchas articulaciones y las contracciones pueden estar en extensión y en flexión (Blowey y Weaver, 2011). Se debe considerar que si la flexión incluye la articulación del carpo y los terneros la apoyan para desplazarse, aunque se realice la tenotomía, el pronóstico suele ser pobre.

Algunas recomendaciones: si es necesario, recorte las pezuñas para ayudar a la conformación normal y para promover la colocación correcta en el piso. Si no se corrige la alteración con la tenotomía de los tendones, se puede considerar la realización de una desmotomía del ligamento suspensor del menudillo (músculo interóseo).

\section{CONCLUSIÓN}

Se reporta el caso de una CTF severa en un ternero con resolución exitosa y se explica a detalle la historia, tratamientos y el proceso quirúrgico que se llevaron a cabo. Este caso refuerza la literatura médica veterinaria que aborda estos casos, ya que la mayoría de los reportes existentes se han enfocado en casos de contractura leve que son resueltos con fármacos y fisioterapia. Se reporta un tiempo corto de recuperación posquirúrgico debido al uso de una férula adecuada, terapia farmacológica y estimulación de ejercicio físico como terapia de rehabilitación. 


\section{LITERATURA CITADA}

ALSAAOD M, Huber S, Beer G, Kohler P, Schüpbach-Regula G, Steiner A. 2017. Locomotion characteristics of dairy cows walking on pasture and the effect of artificial flooring systems on locomotion comfort. Journal of Dairy Science. 100(10):8330-8337. https://doi.org/10.3168/jds.2017-12760

ARIETA RRJ, Fernandez FJA. 2011. Contractura a nivel de tendones en extremidades anteriores de un becerro: informe de un caso clínico. REDVET. 12(12):1-4. E-ISSN: 16957504. https://www.redalyc.org/pdf/636/63622039011.pdf

BLOWEY RW, Weaver AD. 2011. Diseases and disorders of cattle. 3th ed. China: MosbyElsevier. Pp. 99. ISBN: 978-0-7234-3602-7.

https://books.google.com.mx/books?hl=es\&lr=\&id=roHrW4mc_8kC\&oi=fnd\&pg=PR1\&dq $=$ Diseases + and + disorders + of + cattle. +3 th + ed + blowey\&ots $=$

BRUIJNIS MR, Hogeveen H, Stassen EN. 2010. Assessing economic consequences of foot disorders in dairy cattle using a dynamic stochastic simulation model. Journal of Dairy Science. 93(6):2419-2432. https://doi.org/10.3168/jds.2009-2721

DIVERS TJ, Peek SF. 2017. Rebhun's diseases of dairy cattle. 3rd ed. St. Louis, Missouri: Elsevier Health Sciences - Saunders. Pp. 704. https://doi.org/10.1016/C2013-0-12799-7

DUNCANSON G. 2010. Veterinary treatment for working equines. 2nd ed. London: CABI. Pp. 104. ISBN-13: 978-1-84593-655-6.

https://books.google.com.mx/books?hl=es\&lr=\&id=jBvAQriQJDMC\&oi=fnd\&pg=PR5\&dq $=$ Veterinary+treatment+for+working+equines+2+ed\&ots=

FEITO-HERMIDA J, Jareño-Moreno S, Mínguez-Rico A, Kawiecki-Peralta A. 2013. Retracción de tendones en terneros. REDUCA. 5:195-199. www.revistareduca.es/index.php/reduca/article/view/1632

FERNANDES MEDSL, de Albuquerque-Carvalho L, Chenard MG, Pitombo CA, dos Santos OJ, Caldas SA, Nogueira VA, Helayel MA. 2020. Management of a Congenital Flexural Deformity in a Calf-Surgical and Pathological Aspects. Acta Scientiae Veterinariae. 48(1):581.

https://www.seer.ufrgs.br/ActaScientiaeVeterinariae/article/view/104033

FOSSUM TW. 2019. Cirugía en pequeños animales. 5th ed. España: Elsevier. Pp. 1632. ISBN:978-84-9113-380-3.

https://books.google.com.mx/books?hl=es\&lr=\&id=48nSDwAAQBAJ\&oi=fnd\&pg=PP1\&d $q=$ 
GAUGHAN EM. 2017. Flexural limb deformities of the carpus and fetlock in foals. Veterinary Clinics of North America: Equine Practice. 33:331-42. http://dx.doi.org/10.1016/j.cveq.2017.03.004

KIRKENDALL DT, Garrett WE. 1997. Function and biomechanics of tendons. Scandinavian Journal of Medicine and Science Sports. 7 (2):62-66. https://doi.org/10.1111/j.1600-0838.1997.tb00120.x

PICCIONE G, Casella S, Pennisi P, Giannetto C, Costa A, Caola G. 2010. Monitoring of physiological and blood parameters during perinatal and neonatal period in calves. Arquivo Brasileiro de Medicina Veterinária e Zootecnia. 62(1):1-12. https://doi.org/10.1590/S0102-09352010000100001

SATO A, Kato T, Tajima M. 2020. Flexor tendon transection and post-surgical external fixation in calves affected by severe metacarpophalangeal flexural deformity. Journal of Veterinary Medical Science. 82(10):1480-1483. https://doi.org/10.1292/jvms.20-0057

SOHRT JT, Heppelmann M, Rehage J, Staszyk C. 2013. Tenotomy of carpal and digital flexor tendons for correction of congenital neuromyodysplasia in a calf. Tierarztliche Praxis. 41(2):113-118. https://doi.org/10.1055/s-0038-1623160

STEINER A, Anderson DE, Desrochers A. 2014. Diseases of the Tendons and Tendon Sheaths. Veterinary Clinics: North America - Food Animal Practice. 30(1):157-175. https://doi.org/10.1016/j.cvfa.2013.11.002

TAMILMAHAN P, Prabhakar R. 2018. Surgical management of congenital flexor tendon deformity in calves: A review of three cases. Journal of Entomology and Zoology Studies. 6(3):544-546. http://krishikosh.egranth.ac.in/handle/1/5810139315

WAVREILLE G, Fontaine C. 2009. Tendón normal: anatomía y fisiología. EMC-Aparato Locomotor. 42(1):1-12. https://doi.org/10.1016/S1286-935X(09)70909-8

WEAVER AD, Atkinson O, St. Jean G, Steiner A. 2018. Contracted flexor tendons. In: Weaver D, Atkinson O, Steiner A, St. Jean G. Eds. Bovine Surgery and Lameness. 3rd ed. Oxford: John Wiley and Sons Ltd. Pp. 267. ISBN: 9781119040514. https://books.google.com.mx/books?hl=es\&lr=\&id=UHFODwAAQBAJ\&oi=fnd\&pg=PP2\& $\mathrm{dq}=$ Bovine + Surgery+and+Lameness+3rd\&ots $=$ 